\title{
ARENS PRODUCT AND THE ALGEBRA OF DOUBLE MULTIPLIERS. II
}

PAK-KEN WONG

\begin{abstract}
We show that the algebra of double multipliers of a certain Banach algebra $A$ can be embedded in the second conjugate space $A^{* *}$ of $A$. This generalizes the previous work by the author for $B^{*}$-algebras.
\end{abstract}

1. Introduction. Let $A$ be a semisimple Banach algebra and $M(A)$ the algebra of double multipliers on $A$. The author recently showed that $M(A)$ is isomorphic to ( $\left.A^{* *}, \circ\right)$ if and only if $A$ has the following properties: (1) $A$ is Arens regular, (2) $A$ has an approximate identity, and (3) $\pi(A)$ is an ideal of $\left(A^{* *}, \circ\right)[10$, Theorem, p. 442]. In general, not many Banach algebras possess all these properties. The purpose of this paper is to establish an embedding of $M(A)$ in the second conjugate space $A^{* *}$ of $A$ for a semisimple Banach algebra $A$ with an approximate identity. In fact, we show that $M(A)$ is isometrically isomorphic to $M^{* *} / N^{* *}$, where $M^{* *}=\{F \in$ $A^{* *}: \quad F \circ \pi(x)$ and $\pi(x) \circ F \in \pi(A)$ for all $\left.x \in A\right\}$ and $N^{* *}=\left\{F \in A^{* *}\right.$ : $F(f \circ \pi)=F(x \circ f)=0$ for all $x \in A$ and $\left.f \in a^{*}\right\}$. The main idea of the proof of this result is essentially contained in the proofs of $[8$, Lemma 2.1, p. 80 and 10 , Theorem, p. 422]. This embedding was studied in [1 and 8] for $B^{*}$-algebras.

2. Notation and preliminaries. Definitions not explicitly given are taken from Rickart's book [6].

Let $A$ be a Banach algebra. Then $A^{*}$ and $A^{* *}$ will denote the first and second conjugate spaces of $A$, and $\pi$ the canonical map of $A$ into $A^{* *}$. The two Arens products on $A^{* *}$ are defined in stages according to the following rules (see [2]). Let $x, y \in A, f \in A^{*}$, and $F, G \in A^{* *}$.

Define $f \circ x$ by $(f \circ x)(y)=f(x y)$. Then $f \circ x \in A^{*}$.

Define $G \circ f$ by $(G \circ f)(x)=G(f \circ x)$. Then $G \circ f \in A^{*}$.

Define $F \circ G$ by $(F \circ G)(f)=F(G \circ f)$. Then $F \circ G \in A^{* *}$.

Define $x \circ^{\prime} f$ by $\left(x \circ^{\prime} f\right)(y)=f(y x)$. Then $x \circ^{\prime} f \in A^{*}$.

Define $f \circ^{\prime} F$ by $\left(f \circ^{\prime} F\right)(x)=F\left(x \circ^{\prime} f\right)$. Then $f \circ^{\prime} F \in A^{*}$.

Define $F \circ^{\prime} G$ by $\left(F \circ^{\prime} G\right)(f)=G\left(f \circ^{\prime} F\right)$. Then $F \circ^{\prime} G \in A^{* *}$.

Received by the editors February 25, 1986.

1980 Mathematics Subject Classification (1985 Revision). Primary 46H10; Secondary 46H99.

Key words and phrases. Arens products, Arens regularity, approximate identity, double multiplier, $B^{*}$-algebra, conjugate space.

This research was done while the author was visiting the University of Hong Kong. The author gratefully acknowledges the hospitality of the Department of Mathematics of the University.

(c) 1987 American Mathematical Society $0002-9939 / 87 \$ 1.00+\$ .25$ per page 
$A^{* *}$ is a Banach algebra under the products $F \circ G$ and $F \circ^{\prime} G$ and $\pi$ is an algebra isomorphism of $A$ into $\left(A^{* *}, \circ\right)$ and $\left(A^{* *}, \circ^{\prime}\right)$. In general, $\circ$ and $\circ^{\prime}$ are distinct on $A^{* *}$. If they agree on $A^{* *}$, then $A$ is called Arens regular.

The following properties of the Arens products will be used often in the rest of this paper.

LemMA 2.1. Let $A$ be a Banach algebra. Then for all $x \in A, f \in A^{*}$, and $F, G \in A^{* *}$, we have

(1) $\pi(x) \circ F=\pi(x) \circ F$ and $F \circ \pi(x)=F \circ^{\prime} \pi(x)$.

(2) If $\left\{F_{\beta}\right\} \subset A^{* *}$ and $F_{\beta} \rightarrow F$ weakly in $A^{* *}$, then $F_{\beta} \circ G \rightarrow F \circ G$ and $G \circ$ $F_{\beta} \rightarrow G \circ^{\prime} F$ weakly.

(3) $F(x \circ f)=(F \circ \pi(x))(f)$.

Proof. (1) is proved in [2, p. 843] and (2) in [2, p. 842].

(3) It is easy to see that $\pi(x) \circ f=x \circ^{\prime} f$. Therefore

$$
(F \circ \pi(x))(f)=F(\pi(x) \circ f)=F(x \circ f) .
$$

This completes the proof of the lemma.

We say that a Banach algebra $A$ has an approximate identity if there exists a net $\left\{e_{\alpha}\right\}$ in $A$ such that $\left\|e_{\alpha}\right\| \leqslant 1$ for all $\alpha$ and $x=\lim e_{\alpha} x=\lim x e_{\alpha}$ for all $x \in A$.

Let $A$ be a semisimple Banach algebra. A pair $\left(T_{1}, T_{2}\right)$ of operators from $A$ to $A$ is called a double multiplier (centralizer) on $A$ provided that $x\left(T_{1} y\right)=\left(T_{2} x\right) y$ for all $x, y \in A$. It is knowns that $T_{1}$ and $T_{2}$ are continuous linear operators on $A$ such that $T_{1}(x y)=\left(T_{1} x\right) y$ and $T_{2}(x y)=x\left(T_{2} y\right)$. The set $M(A)$ of all double multipliers on $A$ is a Banach algebra with identity and $A$ can be identified as a two-sided ideal of $M(A)$ (see [4 and 5]).

In this paper, all algebras and spaces under consideration are over the complex field.

3. Extensions of double multipliers. Let $A$ be a semisimple Banach algebra. We show that each double multipler $T=\left(T_{1}, T_{2}\right)$ on $A$ can be extended to a double multiplier on $\left(A^{* *}, \circ\right)$. In fact, for all $x \in A$ and $f \in a^{*}$, we define

$$
\left(f \circ T_{1}\right)(x)=f\left(T_{1} x\right) \text { and }\left(f \circ T_{2}\right)(x)=f\left(T_{2} x\right) .
$$

Then $f \circ T_{1}$ and $f \circ T_{2} \in A^{*}$. For all $F \in A^{* *}$, we define

$$
T_{1}^{\#}(F)(f)=F\left(f \circ T_{1}\right) \quad \text { and } \quad T_{2}^{\#}(F)(f)=F\left(f \circ T_{2}\right)
$$

Then $T_{1}^{\#}(f)$ and $T_{2}^{\#}(F) \in A^{* *}$.

THEOREM 3.1. Let $A$ be a semisimple Banach algebra. Then for each $T=\left(T_{1}, T_{2}\right) \in$ $M(A), T^{\#}=\left(T_{1}^{\#}, T_{2}^{\#}\right)$ is a continuous double multiplier on $\left(A^{* *},{ }^{\circ}\right)$ and $T^{\#} \mid \pi(A)$ $=T$. 
Proof. Let $F$ and $G \in A^{* *}$. Then by Goldstine's Theorem, there exist nets $\left\{x_{m}\right\}$ and $\left\{y_{n}\right\}$ in $A$ such that $\pi\left(x_{m}\right) \rightarrow F$ and $\pi\left(y_{n}\right) \rightarrow G$ weakly in $A^{* *}$. Then for all $f \in A^{*}$, we have

$$
\begin{aligned}
\left(F \circ T_{1}^{\#}(G)\right)(f) & =\lim _{m}\left(T_{1}^{\#}(G) \circ f\right)\left(x_{m}\right)=\lim _{m} G\left(\left(f \circ x_{m}\right) \circ T_{1}\right) \\
& =\lim _{m} \lim _{n}\left(\left(f \circ x_{m}\right) \circ T_{1}\right)\left(y_{n}\right) \\
& =\lim _{m} \lim _{n} f\left(\left(T_{2} x_{m}\right) y_{n}\right)=\lim _{m} G\left(f \circ T_{2} x_{m}\right) \\
& =\lim _{m}\left((G \circ f) \circ T_{2}\right)\left(x_{m}\right)=\left(T_{2}^{\#}(F) \circ G\right)(f) .
\end{aligned}
$$

Therefore $F \circ T_{1}^{\#}(G)=T_{2}^{\#}(F) \circ G$. Similarly, we can show that $T_{1}^{\#}(F \circ G)=$ $T_{1}^{\#}(F) \circ G$ and $T_{2}^{\#}(F \circ G)=F \circ T_{2}^{\#}(G)$. It is easy to see that $\left\|T_{1}^{\#}\right\| \leqslant\left\|T_{1}\right\|$ and $\left\|T_{2}^{\#}\right\| \leqslant\left\|T_{2}\right\|$. Therefore $\left\|T^{\#}\right\| \leqslant\|T\|$. Since $T_{1}^{\#}(\pi(x))=\pi\left(T_{1} x\right)$ and $T_{2}^{\#}(\pi(x))=$ $\pi\left(T_{2} x\right)$, we have $T^{\#} \mid \pi(A)=T$ and the theorem is proved.

4. The subalgebras $M^{* *}$ and $N^{* *}$ of $\left(A^{* *},{ }^{\circ}\right)$. For any Banach algebra $A$, set

$$
M^{* *}=\left\{F \in A^{* *}: F \circ \pi(x) \text { and } \pi(x) \circ F \in \pi(A) \text { for all } x \in A\right\}
$$

and

$$
N^{* *}=\left\{F \in A^{* *}: F(f \circ x)=F\left(x \circ^{\prime} f\right)=0 \text { for all } x \in A \text { and } f \in A^{*}\right\} .
$$

Then $\pi(A)$ is a two-sided ideal of $\left(A^{* *}, \circ\right)$ if and only if $M^{* *}=A^{* *}$.

Lemma 4.1. Let $A$ be a Banach algebra. Then $M^{* *}$ and $N^{* *}$ are closed subalgebras of $\left(A^{* *}, \circ\right)$ and $\left(A^{* *}, \circ^{\prime}\right)$. Also $N^{* *}$ is a two-sided ideal in $M^{* *}$ and $N^{* *}=\{F \in$ $\left.A^{* *}: A^{* *} \circ F=F \circ^{\prime} A^{* *}=(0)\right\}$.

Proof. For any $F \in N^{* *}$, easy calculations show that $\pi(A) \circ F=(0)$ and $F \circ \pi(A)=(0)$. Hence $A^{* *} \circ F=F \circ^{\prime} A^{* *}=(0)$ and so $N^{* *}=\left\{F \in A^{* *}: A^{* *} \circ F\right.$ $\left.=F \circ^{\prime} A^{* *}=(0)\right\}$. Thus if $F, G \in N^{* *}$, then $\pi(A) \circ(F \circ G)=(F \circ G) \circ \pi(A)=(0)$ and so $F \circ G \in N^{* *}$. Similarly, $F \circ^{\prime} G \in N^{* *}$. It is easy to check that $M^{* *}$ and $N^{* *}$ are closed subalgebras in $\left(A^{* *}, \circ^{\circ}\right)$ and $\left(A^{* *}, \circ^{\prime}\right)$ and $N^{* *}$ is a two-sided ideal of $M^{* *}$. Therefore the lemma is proved.

In the rest of this section, $A$ will be a semisimple Banach algebra with an approximate identity $\left\{e_{\alpha}\right\}$. It follows from [3, Proposition 7, p. 146] that $\left(A^{* *},{ }^{\circ}\right)$ has a right identity $I$. Also $I$ is a left identity for $\left(A^{* *}, \circ^{\prime}\right)$.

THEOREM 4.2. Let $A$ be a semisimple Banach algebra with an approximate identity $\left\{e_{\alpha}\right\}$. Then the Arens products $\circ$ and $\circ^{\prime}$ agree on $M^{* *} / N^{* *}$ and $M^{* *} / N^{* *}$ is a Banach algebra with an identity.

Proof. Let $F, G \in M^{* *}, f \in A^{*}$, and $x \in A$. Then by Cohen's Factorization Theorem [3, Theorem 10, p. 61] $x=y z$ with $y, z \in A$. Hence

$$
\begin{aligned}
(F \circ G-F \circ G)(f \circ x) & =(F \circ G-F \circ G)((f \circ y) \circ z) \\
& =(\pi(z) \circ F \circ G-\pi(z) \circ F \circ G)(f \circ y)=0 .
\end{aligned}
$$


Similarly, $\left(F \circ G-F \circ^{\prime} G\right)\left(x \circ^{\prime} f\right)=0$. Therefore $F \circ G-F \circ^{\prime} G \in N^{* *}$ and so $\circ$ and $\circ^{\prime}$ agree on $M^{* *} / N^{* *}$. Since $I$ is a right identity for $\left(A^{* *},{ }^{\circ}\right)$ and a left identity for $\left(A^{* *}, \circ\right)$ and $I \in M^{* *}$, it follows that $M^{* *} / N^{* *}$ is a Banach algebra with an identity.

REMARK. For a certain Banach algebra $A, N^{* *}$ is the radical of $\left(A^{* *}, \circ\right)$ (for example, see [9, Theorem 4.1, p. 440]).

The following result is essentially contained in the proof of [8, Lemma 2.1, p. 80]. It is useful in the next section.

LEMMA 4.3. Let $A$ be a semisimple Banach algebra with an approximate identity $\left\{e_{\alpha}\right\}, T=\left(T_{1}, T_{2}\right) \in M(A)$, and $x \in A$. Then

(1) $T_{1}^{\#}(I) \circ \pi(x)=T_{2}^{\#}(I) \circ \pi(x)=\pi\left(T_{1} x\right)$.

(2) $\pi(x) \circ T_{1}^{\#}(I)=\pi(x) \circ T_{2}^{\#}(I)=\pi\left(T_{2} x\right)$.

(3) $T_{1}^{\#}(I)=T_{2}^{\#}(I)+N$ for some $N \in N^{* *}$.

(4) If $T_{1}^{\#}(I)$ and $T_{2}^{\#}(I)$ are both in $N^{* *}$, then $T=0$.

Proof. (1) For all $f \in A^{*}$, we have

$$
\begin{aligned}
\left(T_{1}^{\#}(I) \circ \pi(x)\right)(f) & =I\left((\pi(x) \circ f) \circ T_{1}\right)=\lim _{\alpha}\left((\pi(x) \circ f) \circ T_{1}\right)\left(e_{\alpha}\right) \\
& =\lim _{\alpha} f\left(T_{1}\left(e_{\alpha} x\right)\right)=\lim _{\alpha}\left(f \circ T_{1}\right)\left(e_{\alpha} x\right) \\
& =\left(f \circ T_{1}\right)(x)=f\left(T_{1} x\right) .
\end{aligned}
$$

Hence $T_{1}^{\#}(I) \circ \pi(x)=\pi\left(T_{1} x\right)$. Similarly, $T_{2}^{\#}(I) \circ \pi(x)=\pi\left(T_{1} x\right)$. Therefore $(1)$ is proved. Similarly we can prove (2).

(3) Let $N=T_{1}^{\#}(I)-T_{2}^{\#}(I)$. Then

$$
\begin{aligned}
N(f \circ x) & =\pi(x)(N \circ f)=(\pi(x) \circ N)(f) \\
& =\left(\pi(x) \circ T_{1}^{\#}(I)-\pi(x) \circ T_{2}^{\#}(I)\right)(f) \\
& =\left(\pi\left(T_{2} x\right)-\pi\left(T_{2} x\right)\right)(f)=0 .
\end{aligned}
$$

Similarly, $N\left(x \circ^{\prime} f\right)=0$. Therefore $N \in N^{* *}$ and so $T_{1}^{\#}(I)=T_{2}^{\#}(I)+N$.

(4) Since $T_{1}^{\#}(I)$ and $T_{2}^{\#}(I) \in N^{* *}$, we have

$$
\pi\left(T_{1} x\right)(f)=\left(T_{2}^{\#}(I) \circ \pi(x)\right)(f)=T_{2}^{\#}(I)(x \circ f)=0 .
$$

Hence $T_{1} x=0$ and so $T_{1}=0$. Also

$$
\pi\left(T_{2} x\right)(f)=\left(\pi(x) \circ T_{1}^{\#}(I)\right)(f)=T_{1}^{\#}(I)(f \circ x)=0 .
$$

Therefore $T_{2}=0$ and so $T=0$. This completes the proof of the lemma.

Notation. In the rest of this paper, for each $F \in M^{* *}$, we write $\tilde{F}=F+N^{* *}$.

5. The algebras $M(A)$ and $M^{* *} / N^{* *}$. We have the main result of this paper.

THEOREM 5.1. Let $A$ be a semisimple Banach algebra with an approximate identity $\left\{e_{\alpha}\right\}$. Then $M(A)$ is isometrically isomorphic to $M^{* *} / N^{* *}$.

Proof. Let $T=\left(T_{1}, T_{2}\right) \in M(A)$. Then by Lemma 4.3, $T_{1}^{\#}(I)$ and $T_{2}^{\#}(I) \in M^{* *}$ and $\widetilde{T_{1}^{\#}(I)}=\widetilde{T_{2}^{\#}(I)}$. We define a mapping $\Phi$ from $M(A)$ to $M^{* *} / N^{* *}$ by

$$
\Phi(T)=\widetilde{T_{1}^{\#}(I)}=\widetilde{T_{2}^{\#}(I)} .
$$


We show that $\Phi$ is an isometric isomorphism from $M(A)$ onto $M^{* *} / N^{* *}$. It is clear that $\Phi$ is linear. Let $x \in A$ and $f \in A^{*}$. If $\Phi(T)=0$, then $T_{1}^{\#}(I)$ and $T_{2}^{\#}(I)$ are both in $N^{* *}$. Hence by Lemma 4.3, T=0. Therefore $\Phi$ is one-one. Let $S=\left(S_{1}, S_{2}\right) \in M(A)$. Since $S T=\left(S_{1} T_{1}, T_{2} S_{2}\right)$, by Lemma 4.3, we have

$$
\begin{aligned}
\left(S_{1}^{\#}(I) \circ T_{1}^{\#}(I)\right)(f \circ x) & =\left(\pi(x) \circ S_{1}^{\#}(I) \circ T_{1}^{\#}(I)\right)(f) \\
& =\left(\pi\left(S_{2} x\right) \circ T_{1}^{\#}(I)\right)(f)=\pi\left(T_{2} S_{2} x\right)(f) \\
& =\left(\pi(x) \circ\left(S_{1} T_{1}\right)^{\#}(I)\right)(f)=\left(S_{1} T_{1}\right)^{\#}(I)(f \circ x) .
\end{aligned}
$$

Similarly, we have

$$
\left(S_{1}^{\#}(I) \circ T_{1}^{\#}(I)\right)(x \circ f)=\left(S_{1} T_{1}\right)^{\#}(I)(x \circ f) .
$$

Therefore $\Phi(S T)=\Phi(S) \Phi(t)$ and so $\Phi$ is an isomorphism. Let $F \in M^{* *}$. Define

$$
P_{1}(x)=F \circ \pi(x) \quad \text { and } \quad P_{2}(x)=\pi(x) \circ F \quad(x \in A) .
$$

Since $F \in M^{* *}, P=\left(P_{1}, P_{2}\right) \in M(A)$. Also

$$
\begin{aligned}
P_{1}^{\#}(I)(f \circ x) & =I\left((f \circ x) \circ P_{1}\right)=\lim _{\alpha}\left((f \circ x) \circ P_{1}\right)\left(e_{\alpha}\right) \\
& =\lim _{\alpha} f\left(x\left(F \circ \pi\left(e_{\alpha}\right)\right)\right)=\lim _{\alpha} f\left((\pi(x) \circ F) e_{\alpha}\right) \\
& =\lim _{\alpha} f\left(e_{\alpha}(\pi(x) \circ F)\right)=\lim _{\alpha} f\left(\pi\left(e_{\alpha} x\right) \circ F\right) \\
& =\lim _{\alpha}\left(\pi\left(e_{\alpha} x\right) \circ F\right)(f)=\lim _{\alpha} \pi\left(e_{\alpha} x\right)(F \circ f) \\
& =(F \circ f)(x)=F(f \circ x) .
\end{aligned}
$$

Similarly, we have $P_{1}^{\#}(I)\left(x \circ^{\prime} f\right)=F\left(x \circ^{\prime} f\right)$. Therefore $\Phi(P)=\widetilde{P_{1}^{\#}}=\tilde{F}$. Hence $\Phi$ is an onto mapping. It is easy to see that $\|\Phi(T)\| \leqslant\|T\|$. Since $\left\|T_{1}\right\|=\sup \left\{\left|f\left(T_{1} x\right)\right|\right.$ : $\|x\| \leqslant 1,\|f\| \leqslant 1$ for all $x \in A$ and $\left.f \in A^{*}\right\}$, for given $\varepsilon>0$, there exists $y \in A$ and $g \in A^{*}$ with $\|y\| \leqslant 1$ and $\|g\| \leqslant 1$ such that $\left|g\left(T_{1} y\right)\right| \geqslant\left\|T_{1}\right\|-\varepsilon$. Then

$$
\begin{aligned}
I\left((y \circ g) \circ T_{1}\right) & =\lim _{\alpha}(y \circ g)\left(T_{1} e_{\alpha}\right)=\lim _{\alpha} g\left(\left(T_{1} e_{\alpha}\right) y\right)=\lim _{\alpha} g\left(T_{1}\left(e_{\alpha} y\right)\right) \\
& =\lim _{\alpha}\left(g \circ T_{1}\right)\left(e_{\alpha} y\right)=\left(g \circ T_{1}\right)(y)=g\left(T_{1} y\right) .
\end{aligned}
$$

Hence for all $N \in N^{* *}$, we have

$$
\begin{aligned}
\left\|T_{1}^{\#}(I)+N\right\| & \geqslant\left|\left(T_{1}^{\#}(I)+N\right)\left(y \circ^{\prime} g\right)\right|=\left|T_{1}^{\#}(I)\left(y \circ^{\prime} g\right)\right| \\
& =\left|I\left((y \circ g) \circ T_{1}\right)\right|=\left|g\left(T_{1} y\right)\right| \geqslant\left\|T_{1}\right\|-\varepsilon .
\end{aligned}
$$

Since $\varepsilon$ is arbitrary, we have

$$
\|\Phi(T)\|=\left\|\widetilde{T_{1}^{\#}(I)}\right\| \geqslant\left\|T_{1}\right\| .
$$

Similarly, we can show that $\|\Phi(T)\| \geqslant\left\|T_{2}\right\|$ and so $\|\Phi(T)\| \geqslant\|T\|$. Therefore $\|\Phi(T)\|=\|T\|$. This completes the proof of the theorem.

COROllary 5.2. Let $A$ be as in Theorem 5.1. If $\left(A^{* *},{ }^{\circ}\right)$ has an identity $I$, then $N^{* *}=(0)$ and so $M(A)$ is isometrically isomorphic to $M^{* *}$. 
Proof. Let $N \in N^{* *}$. Then for all $f \in A^{*}$, we have

$$
N(f)=(I \circ N)(f)=\lim _{\alpha}\left(\pi\left(e_{\alpha}\right) \circ N\right)(f)=\lim _{\alpha} N\left(f \circ e_{\alpha}\right)=0 .
$$

Therefore $N^{* *}=(0)$ and the corollary follows from Theorem 5.1.

Corollary 5.3. Let $A$ be as in Theorem 5.1. If $A$ is Arens regular, then $M(A)$ is isometrically isomorphic to $M * *$.

Proof. Since $A$ is Arens regular, $\left(A^{* *},{ }^{\circ}\right)$ has an identity $I$. Therefore the result follows from Corollary 5.2.

We now have a slight improvement of [10, Theorem, p. 442].

THEOREM 5.4. Let $A$ be a semisimple Banach algebra. Then $M(A)$ is isometrically isomorphic to $\left(A^{* *}, \circ\right)$ if and only if $A$ has the following properties:

(1) $A$ is Arens regular.

(2) $A$ has an approximate identity.

(3) $\pi(A)$ is a two-sided ideal of $\left(A^{* *}, \circ\right)$.

Proof. Suppose that $M(A)$ is isometrically isomorphic to $\left(A^{* *}, \circ\right)$. Then by Theorem 5.1, we have $N^{* *}=(0)$ and $M^{* *}=A^{* *}$. Hence $\pi(A)$ is a two-sided ideal of $\left(A^{* *}, \circ\right)$. By Theorem $4.2, A$ is Arens regular and $\left(A^{* *}, \circ\right)$ has an identity. Hence it follows from [3, Proposition 7, p. 147] that $A$ has an approximate identity. Therefore $A$ has properties (1), (2), and (3).

Conversely, suppose that $A$ has properties (1), (2), and (3). Then $M^{* *}=A^{* *}$ and so by Corollary $5.3, M(A)$ is isometrically isomorphic to $\left(A^{* *},{ }^{\circ}\right)$. This completes the proof of the theorem.

6. Banach*-algebras. Let $A$ be a Banach*-algebra with a continuous involution. For all $x \in A, f \in A^{*}$, and $F \in A^{* *}$, we define

$$
f^{*}(x)=\overline{f\left(x^{*}\right)} \text { and } F^{*}(f)=\overline{F\left(f^{*}\right)},
$$

where the bar denotes the complex conjugation. Then $f^{*} \in A$ and $F^{*} \in A^{* *}$. If $A$ is a $B^{*}$-algebra, then $\left(A^{* *},{ }^{\circ}\right)$ is a $B^{*}$-algebra under the involution $F \rightarrow F^{*}$ (see [7, p. 192]).

LeMma 6.1. Let $A$ be a Banach *-algebra with a continuous involution. Then

(1) For all $F$ and $G \in A^{* *}$, we have

$$
(F \circ G)^{*}=G^{*} \circ^{\prime} F^{*} \text { and }(F \circ G)^{*}=G^{*} \circ F^{*} \text {. }
$$

(2) $A$ is Arens regular if and only if $\left(A^{* *}, \circ\right)$ is $a^{*}$-algebra.

Proof. (1) For all $x \in A$ and $F \in A^{*}$, it is easy to show that $f^{* \circ} x=\left(x^{*} \circ f\right)^{*}$ and so $G \circ f^{*}=\left(f \circ \circ^{\prime}\right)^{*}$. Then

$$
\begin{aligned}
(F \circ G)^{*}(f) & =\overline{F\left(G \circ f^{*}\right)}=\overline{F\left(\left(f \circ{ }^{\prime} G^{*}\right)^{*}\right)} \\
& =F^{*}\left(f \circ G^{*}\right)=\left(G^{*} \circ F^{*}\right)(f) .
\end{aligned}
$$

Hence $(F \circ G)^{*}=G^{*} \circ^{\prime} F^{*}$. Similarly, we have $(F \circ G)^{*}=G^{*} \circ F^{*}$. 
(2) If $A$ is Arens regular, then it follows from (1) that $\left(A^{* *}, \circ\right)$ is a ${ }^{*}$-algebra. Conversely, suppose that $\left(A^{* *},{ }^{\circ}\right)$ is a ${ }^{*}$-algebra. Then

$$
F \circ G=(F \circ G)^{* *}=\left(G^{*} \circ F^{*}\right)^{*}=F^{* *} \circ^{\prime} G^{* *}=F \circ G .
$$

Hence $A$ is Arens regular and the lemma is proved.

If $A$ is a semisimple Banach *-algebra and $T=\left(T_{1}, T_{2}\right) \in M(A)$, then $T=$ $\left(T_{1}, T_{2}\right) \rightarrow T^{*}=\left(T_{1}^{*}, T_{2}^{*}\right)$ is a continuous involution on $M(A)$, where $T_{i}^{*}(x)=$ $\left(T_{i}\left(x^{*}\right)\right)^{*}(i=1,2)$.

TheOrem 6.2. Let $A$ be a semisimple Banach*-algebra. If $A$ is Arens regular and $A$ has an approximate identity $\left\{e_{\alpha}\right\}$ with $e_{\alpha}^{*}=e_{\alpha}$, then $M(A)$ is isometrically *-isomorphic to $M^{* *}$.

Proof. By Corollary 5.3, $M(A)$ is isometrically isomorphic to $M^{* *}$. By Lemma $6.1,\left(A^{* *}, \circ\right)$ is a *-algebra and so $M^{* *}$ is a closed *-subalgebra of $\left(A^{* *}, \circ\right)$. Let $T=\left(T_{1}, T_{2}\right) \in M(A)$. Then, for all $f \in A^{*}$, we have

$$
\begin{aligned}
\left(T_{1}^{*}\right)^{\#}(I)(f) & =\lim _{\alpha}\left(f \circ T_{1}^{*}\right)\left(e_{\alpha}\right)=\lim _{\alpha} f\left(T_{1}^{*} e_{\alpha}\right) \\
& =\lim _{\alpha} f\left(\left(T_{1}\left(e_{\alpha}\right)\right)^{*}\right)=\lim _{\alpha} \overline{\left(f^{*} \circ T_{1}\right)\left(e_{\alpha}\right)}=\overline{I\left(f^{*} \circ T_{1}\right)} \\
& =T_{1}^{\#}(I)\left(f^{*}\right)=\left(T_{1}^{\#}(I)\right)^{*}(f) .
\end{aligned}
$$

Hence $\left(T_{1}^{*}\right)^{\#}(I)=\left(T_{1}^{\#}(I)\right)^{*}$. Therefore $\Phi\left(T^{*}\right)=(\Phi(T))^{*}$. This completes the proof of the theorem.

If $A$ is a $B^{*}$-algebra, then $A$ is Arens regular and $A$ has an approximate identity. Therefore we have the following result:

Corollary 6.3. If $A$ is a $B^{*}$-algebra, then $M(A)$ is isometrically *-isomorphic to $M^{* *}$.

REMARK. Corollary 6.3 is [8, Lemma 2.1, p. 80$]$.

\section{REFERENCES}

1. C. A. Akemann, G. K. Pedersen, and J. Tomiyama, Multipliers of $C^{*}$-algebras, J. Funct. Anal. 13 (1973), 277-301.

2. R. F. Arens, The adjoint of a bilinear operation, Proc. Amer. Math. Soc. 2 (1951), 839-848.

3. F. F. Bonsall and J. Duncan, Complete normed algebras, Springer, Berlin, 1973.

4. R. C. Busby, Double centralizers and extensions of $C^{*}$-algebras, Trans. Amer. Math. Soc. 132 (1968), 79-99.

5. B. E. Johnson, An introduction to the theory of centralizers, Proc. London Math. Soc. 14 (1964), $299-320$.

6. C. E. Rickart, General theory of Banach algebras, University Series in Higher Math., Van Nostrand, Princeton, N. J., 1960.

7. M. Tomita, The second dual of a $C^{*}$-algebra, Mem. Fac. Sci. Kyushu Univ. Ser. A 21 (1967), $185-193$.

8. P. K. Wong, On the Arens product and annihilator algebras, Proc. Amer. Math. Soc. 30 (1971), 79-83.

9. $437-448$

10. $441-444$. , On the Arens product and certain Banach algebras, Trans. Amer. Math. Soc. 180 (1973),

Department of Mathematics, Seton Hall University, South Orange, New Jersey 07079 